\title{
ePHENIX at eRHIC
}

\section{Klaus DEHMELT*广}

(Stony Brook University USA)

E-mail: klaus.dehmeltestonybrook.edu

eRHIC is a proposed high luminosity, polarized Electron-Ion Collider (EIC), which would make use of the existing RHIC infrastructure. The eRHIC design is based on using one of the two RHIC hadron rings and a multi-pass Energy Recovery Linac (ERL). A polarized electron beam with an energy up to $30 \mathrm{GeV}$ would collide with a number of ion species accelerated in the existing RHIC accelerator complex, from polarized protons with a top energy of $250 \mathrm{GeV}$ to fully-stripped uranium ions with energies up to $100 \mathrm{GeV} / \mathrm{u}$ covering a center-of-mass energy range from 45 to $175 \mathrm{GeV}$ for polarized e+p, and from 32 to $110 \mathrm{GeV}$ for e+A (for large A) collisions. eRHIC will be able to provide electron-hadron collisions in up to three interaction regions. In one of these regions will be the upgraded PHENIX detector, ePHENIX that evolves from the sPHENIX detector currently being designed. It is envisioned to fully use the sPHENIX detector at midrapidities $(|\eta|<1)$, followed by an sPHENIX-Forward upgrade with additional modifications specific to ePHENIX. The following will describe the ePHENIX approach and goals and requirements for day-1 physics at eRHIC.

XXI International Workshop on Deep-Inelastic Scattering and Related Subjects 22-26 April, 2013

Marseilles, France

\footnotetext{
*Speaker.

On behalf of the PHENIX collaboration.
} 

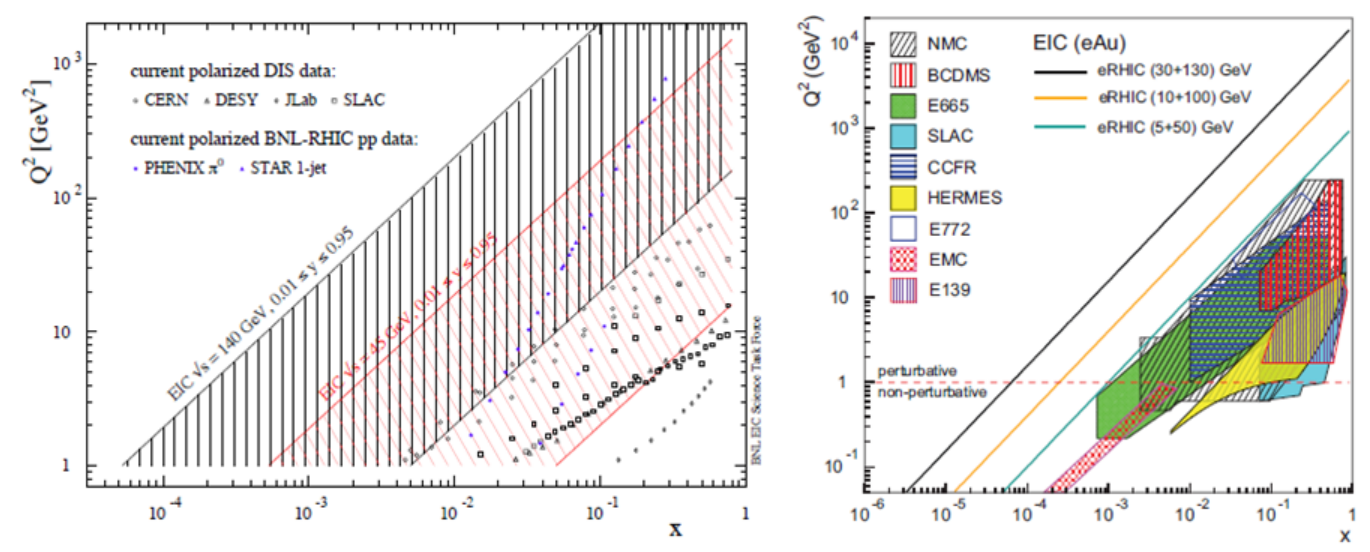

Figure 1: Kinematic range for the EIC. Left: $\mathrm{x}-\mathrm{Q}^{2}$ range that will be covered by eRHIC in Stage 1 for $e+p$ collisions is depicted by the red line. Right: $\mathrm{x}-\mathrm{Q}^{2}$ range that will be covered by eRHIC in Stage 1 for $e+A u$ collisions is depicted by the yellow line. [1]

\section{Introduction}

Many questions are still unanswered when it comes to understanding the role of gluons and sea quarks in QCD. For answering these questions the U.S. community is considering to develop and build a high energy, high luminosity polarized proton-electron and ion-electron collider EIC. Two possible scenarios are under consideration: 1.) eRHIC, and 2.) MEIC.

For 1.) it is foreseen to add a 5-30 GeV polarized electron beam facility to the existing RHIC [1] facility, whereas for 2.) the scenario would be adding a $20-100 \mathrm{GeV}$ proton facility to the existing CEBAF [1] facility, which also will allow to accelerate ions up to $40 \mathrm{GeV} / \mathrm{u}$.

eRHIC will be able to provide electron-hadron collisions in up to three interaction regions and one of these regions will be the upgraded PHENIX detector: ePHENIX. This upgraded detector will evolve from the sPHENIX detector which is currently being designed.

The construction proposal of eRHIC is based on staging. The first stage will include an Energy Recovering Linac (ERL) [1] and will provide electrons in the energy range of 5-10 GeV. These electrons will be colliding with polarized protons in the energy range of $100-250 \mathrm{GeV}$ as well as a wide range of nuclei species $(A l, S i, C u, A u, U)$ and polarized deuterium and ${ }^{3} \mathrm{He}$. The kinematic reach of such a machine can be seen in Fig. 1.

\section{Physics Goals of the EIC}

The EIC will be investigating the nucleon structure; QCD in nuclei; searching for physics beyond the Standard Model (BSM). eRHIC with Stage 1 will be able to make important measurements in these areas but BSM. The studies for the ePHENIX detector were performed with the PHENIX collaboration by the ePHENIX task force [2].

Deep inelastic scattering (DIS) is the process that allows to probe and eventually understand the role of gluons and sea quarks in QCD. This can be accomplished through precision measurements of the structure of protons and nuclei and the dynamics of the partons inside them. DIS probes the target (the hadron) by exchanging (virtual) photons with the target and requires the measurement of 
the angle and energy of the scattered electron. This fully determines two relevant kinematic variables: $Q^{2}$ as the virtuality of the exchanged photon, and $x$ as the momentum fraction of the probed parton. For large $Q^{2}$ the exchange of $\mathrm{W}$ and $\mathrm{Z}$ bosons contributes and the kinematic variables need to be determined by the hadronic final states.

The particular goal is to measure the 3-D structure of the nucleon:

- Gluon and sea quark helicity contributions to nucleon helicity, by means of inclusive and semi-inclusive measurements;

- Quark and gluon transverse momentum distributions (TMD) by means of semi-inclusive measurements (SIDIS);

- The spatial gluon and sea quark distribution in nucleons by means of exclusive measurements (Deeply Virtual Compton Scattering DVCS).

Furthermore, the goal is to investigate QCD in nuclei:

- Nuclear modification of parton distributions by means of small x-physics, $F_{2}^{A}, F_{L}^{A}$ inclusive measurements, and semi-inclusive measurements for flavor-separated structure functions;

- Parton propagation in Cold Nuclear Matter (CNM) by means of semi-inclusive measurements to determine the transport coefficients.

The kinematics for the various measurements and the necessary precision necessitate challenging requirements for tracking, the measurement of the scattered electron, and particle identification (PID). This translates into consideration of momentum and angular resolution, energy measurement and coverage over a wide momentum range respectively.

\section{3. ePHENIX Requirements}

\subsection{Tracking Requirements}

Fig. 2 shows the requirements on tracking for the momentum and angular resolution for a Stage 1 detector at eRHIC. The collision energies shown are for $5 \mathrm{GeV}$ electrons on $100 \mathrm{GeV}$ protons respectively $5 \mathrm{GeV}$ electrons on $250 \mathrm{GeV}$ protons. The requirements are estimated by considering that uncertainties on the yield due to bin migration be held below some acceptable level. It is assumed that a $1 \%$ yield measurement is possible when the total yield uncertainty due to bin shifts is held below $20 \%$.

\subsection{Requirements for Measuring the Scattered Electrons}

Due to the asymmetric beam energy configuration the electron is mainly scattered into central and backward rapidities. Fig. 3 depicts the dependencies of the scattered electrons on the rapidity ranges. Higher $Q^{2}$ and $x$ are selected at central rapidities and smaller $Q^{2}$ at backward rapidities. The energy of the scattered electron varies from zero up to the initial electron energy at backward rapidities and varies in central rapidities even to higher energies.

For identifying the scattered electron it seems to be sufficient to provide satisfactory rejection for $\gamma / \pi$ by means of E/p matching and shower profile determination (Fig. 4). 

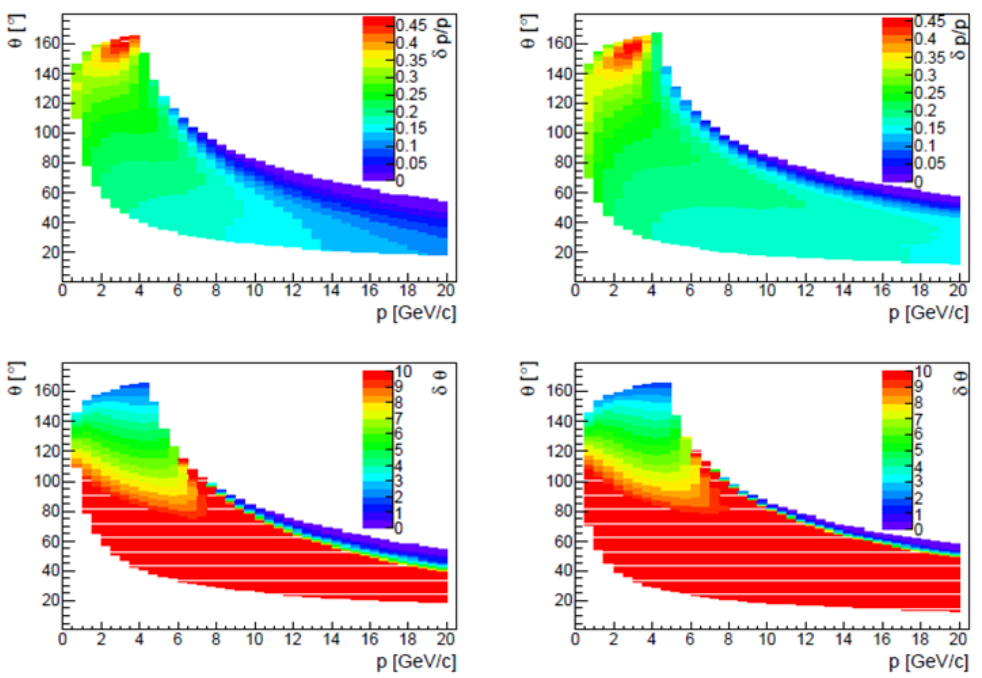

Figure 2: Momentum resolution (top) and angular resolution (bottom) requirements for the ePHENIX detector. [2]
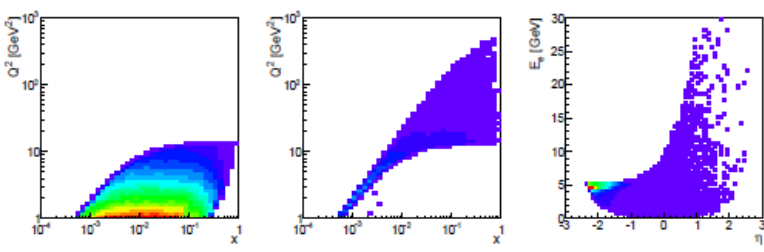

Figure 3: Detected electrons for energies of $5 \mathrm{GeV}$ electrons on $100 \mathrm{GeV}$ protons. Left and Middle: detected electron in backward rapidity $(\eta<-1)$ and midrapidities $(|\eta|<1)$ respectively. Right: scattered electron energies for different pseudorapidities. [2]
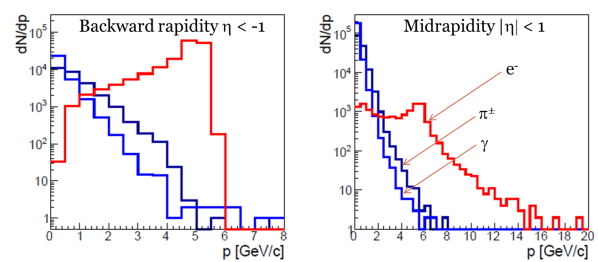

Figure 4: Momentum spectra for electrons (red), charged pions (black) and photons (blue) for $5 \mathrm{GeV}$ electrons on $100 \mathrm{GeV}$ protons. Left: backward rapidity $\eta<-1$. Right: midrapidity $|\eta|<1$. [2]

\subsection{PID Requirements}

Electron identification is required to reconstruct the event kinematics, SIDIS requires pion and Kaon identification for extracting $\Delta s$ and for tagging pions and Kaons in order to measure the transverse spin structure of the proton as well as to investigate the flavor dependence of nuclear PDF.

DVCS needs to tag scattered protons which remain in the beam-pipe. For that the usage of roman pots is foreseen.

\section{Evolution of ePHENIX}

sPHENIX which is described elsewhere in these proceedings will evolve in ePHENIX. In addition to the sPHENIX concept a forward extension for SPHENIX will serve as the infrastructure for ePHENIX (Fig. 5). Furthermore it is necessary to equip the backward rapidity region with detectors for performing the measurements of the scattered electron (Fig. 6). 


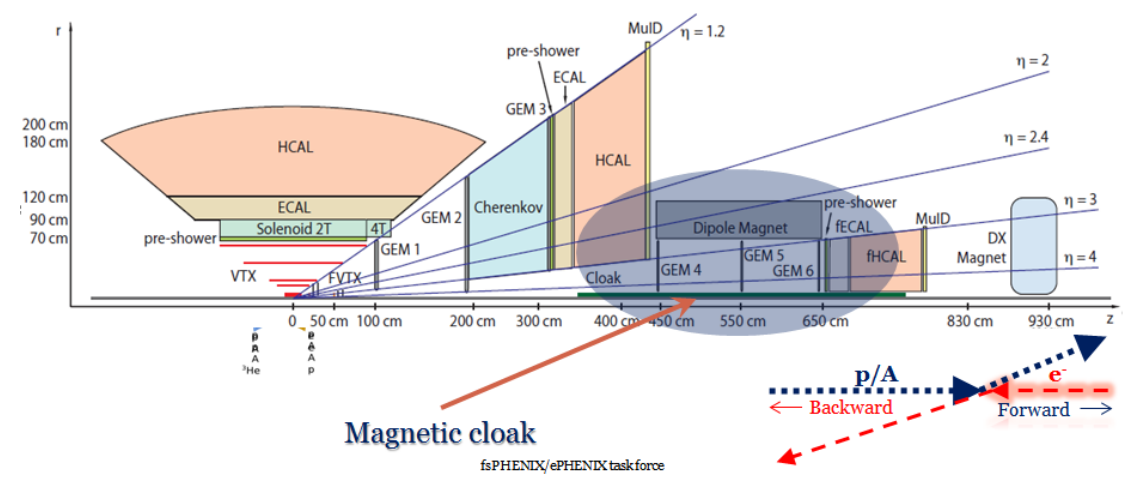

Figure 5: sPHENIX with forward extension. A dipole magnet in the forward region is an option which will require magnetic shielding of the beam pipe (magnetic cloak).

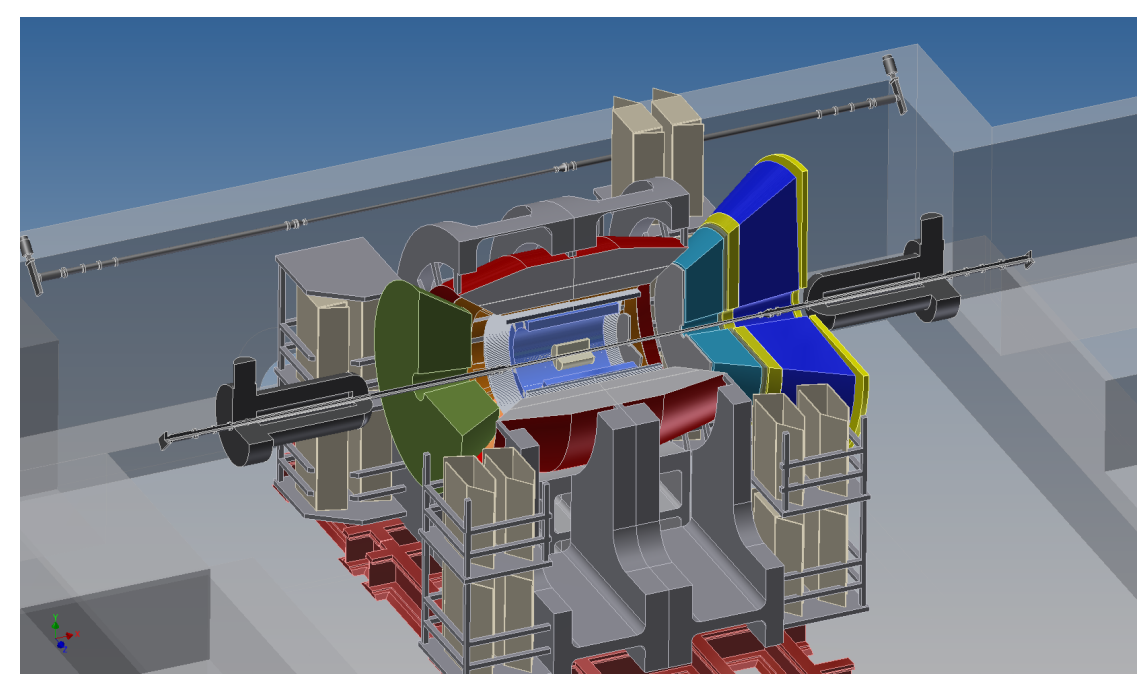

Figure 6: Sketch of the ePHENIX concept. The forward region from sPHENIX is depicted on the right side, the extension for electron measurement on the left side.

\section{Summary}

The conversion of RHIC into an EIC machine, eRHIC is foreseen to happen in a staged approach. The first stage allows to perform measurements from Day 1 with the ePHENIX detector. This detector will be designed for the kinematic and luminosity reach of Stage 1. It involves gradually increasing the electron energy to $10 \mathrm{GeV}$ which allows $\sqrt{s}$ up to $100 \mathrm{GeV}$, and for $\mathscr{L}$ up to $\approx 4 \times 10^{33} \mathrm{~cm}^{-2} \mathrm{~s}^{-1}$.

It is foreseen to make full use of PHENIX upgrades to sPHENIX and Forward sPHENIX with additional modification to evolve into ePHENIX.

\section{References}

[1] A. Accardi et al., Electron Ion Collider: The Next QCD Frontier - Understanding the glue that binds us all, arXiv:1212.1701[nucl-ex]

[2] A. Adare et al., An Upgrade Concept from the PHENIX Collaboration, arXiv:1207.6378[nucl-ex]. 